\title{
Experimental Study of Starvation and Flow Behavior in Grease-Lubricated EHD Contact
}

\author{
Jing Chen $^{1)^{*}}$, Hiroyoshi Tanaka ${ }^{2)}$ and Joichi Sugimura ${ }^{3)}$ \\ ${ }^{1)}$ Graduate School of Engineering, Kyushu University \\ 744 Motooka, Nishi-ku, Fukuoka 819-0395, Japan \\ ${ }^{2)}$ Department of Mechanical Engineering, Kyushu University \\ 744 Motooka, Nishi-ku, Fukuoka 819-0395, Japan \\ ${ }^{3)}$ International Institute for Carbon-neutral Energy Research, Kyushu University \\ 744 Motooka, Nishi-ku, Fukuoka 819-0395, Japan \\ *Corresponding author: 3TE12064K@s.kyushu-u.ac.jp
}

( Manuscript received 11 October 2014; accepted 9 December 2014; published 28 February 2015 )

\begin{abstract}
This paper describes an experimental study of starvation and flow behavior in grease-lubricated elastohydrodynamic contact. Rolling tests were conducted with four grease samples with different thickeners and base oils in a conventional ball-on-disk test rig. The EHL central film thickness was determined with SLIM, the Spacer Layer Imaging Method, and grease flow around the conjunction and the flow pattern on the track of the disk specimen were observed with CCD cameras. The grease track included some thickener deposited on the track and a corrugated fingerlike flow pattern outward from the center of the track. It was found that the flow pattern varied with grease type and test conditions, and that the average interval between fingers decreased with the entrainment speed. At higher speeds, inlet starvation occurred and the finger pattern was deformed and gradually ruptured. The speed of the finger-loss was higher than the speed at which starvation started. It was also found that the starvation speed was greater with greases having lower apparent viscosity at lower shear rate and higher apparent viscosity at high shear rate. This implied that the replenishment and entrainment of the greases depended on the viscosity characteristics of the greases.
\end{abstract}

Keywords: grease, elastohydrodynamic lubrication, starvation, viscosity, film thickness, flow pattern

\section{Introduction}

Grease is a semi-solid consisting of thickener dispersed in base oil. It is widely applied in machine elements such as bearings, gears and joints. Lubricating greases keep their performance without resupply or replacement for a long period of time under proper conditions. However, the lubrication mechanism of greases is still not fully understood [1]. The behaviors of thickener [2,3], and resulting complex rheological properties of grease makes it almost impossible to make accurate prediction of the film thickness or the friction coefficient based on the existing hydrodynamic and EHL theories.

One of the problems in grease lubrication is its limited performance at high speeds. The main cause for this is that once the grease is pushed aside by the passage of a rolling element during the initial phase of operation, the low mobility of grease prevents it from turning back to the contact point easily. It often leads to depletion of lubricant to maintain a film separating the interacting surfaces, and may ultimately lead to the failure and damage. At present, there is no consensus on the mechanisms of film formation process in the starved regime, and on the influence of constituents of grease on lubrication performance.

In the starved conditions, the film formation is determined by the quantity of lubricant supplied to the contact. The most widely used mechanism is the sponge model [4]. When sufficient force to shear the grease is applied, the grease will release base oil, which lubricates the contact zone. However, the oversimplified assumption often does not provide a good prediction that agrees with the experimental observation. The film thickness is generally thinner than the fully-flooded base oil film thickness. The rate at which lubricant is entrained and the rate at which it is lost around the contact, i.e. the feed and loss rates have been deemed as the determining factors for the degree of starvation in grease lubrication [5]. Thus the feed mechanism in the starved regime has become particularly important. It is necessary to understand not only the entrainment of the 
lubricants into the contact but also the behaviors of lubricants in the space surrounding the contact from which the lubricant is supplied.

The early experimental work on the starved oil regime provide a good start point for further study on the flow behavior of grease in the vicinity of a contact. As early as 1970, Wedeven et al. [6] showed that film thickness in starved elastohydrodynamic contact could be predicted using the inlet distance $S$ which was defined as the distance from the position of the inlet meniscus boundary to the edge of the contact. They observed that the lubricant which escaped from the outlet of conjunction zone grew into cavitation fingers both in oil and grease lubricated systems. Later Pemberton and Cameron [7] using aerated oil revealed the 'butterfly-like' meniscus shape of oil flow surrounding the contact point. Their results showed the inlet distance $S$ decreased as speed increased, and suggested that a new equilibrium would be set up by the change in the feed-loss rate. They proposed that the rate of feed was more affected by the shape of the inlet meniscus rather than speed in starved regime. Several authors [8-10] modeled the feed mechanism by assuming that the interacting surface was covered by a uniform layer of lubricant, and that the ball contact produced a depressed oil layer shape, with two adjacent side oil ridges lying beside the ball. Surface tension and viscosity [8], Van der Waals forces [9] and centrifugal forces [10] were assumed to be the driving forces to draw adjacent oil back to contact zone.

In the case of grease lubrication, thickener network has also to be considered. Cann et al. [11,12] proposed a model that the grease-lubricated contacts are layered by a shear degraded residual thickener layer and the oil layer formed by hydrodynamic action. Kaneta et al. [13] studied the effect of thickener structure of diurea greases on the formation of non-uniform EHL film. Kimura et al. [14] controlled the thickness of the thickener fibers through the optimization of the heating-cooling conditions in its manufacturing process. Yokouchi et al. [15] and Oikawa et al. [16] studied the effect of fiber length, and showed that the grease with longer soap fibers was more easily entrained into the contact and showed favorable lubrication with low friction coefficient. All these works suggest that the role of thickener in grease lubrication should not be neglected.
Åström et al. [17] added molybdenum disulfide particles to the grease for observing the movements and distribution of grease in the vicinity of the contact, and showed that the outlet cavitation opened up and formed the fingerlike track pattern. In their experiment the finger pattern remained unchanged several days after test. Otsu et al. [18] conducted both lubricated point contact sliding tests and separating tests in oil lubrication, and showed that the growth of the cavity pattern depended on the rapid evolution of negative pressure at the exit of the conjunction through a numerical analysis. Similarly, accompanied by the development of cavity at the exit of the conjunction, the negative hydrodynamic pressure gradient triggers the instability and induces the growth of finger pattern outward from the cavity-grease meniscus interface.

In the present study, the effect of lubrication condition on grease flow pattern in a ball-on-disk EHL contact is investigated. The purpose of this study is to understand the grease feed mechanisms for film formation in the starved regime.

\section{Experimental}

\subsection{Materials}

Four grease samples with different thickener, base oil and its viscosity were chosen for this study. Samples L1 to L3 and D consisted of only base oil and thickener without any additives. Their composition is given in Table 1.

2.2 Film Thickness Measurement and EHL Track Observation

Film thickness measurement and EHL track pattern observation were conducted in a ball-on-disk test rig. A schematic illustration of the rig is shown in Fig. 1. The disk is made of BK7 glass coated with chromium and silica overlay. The ball is made of JIS SUJ2 steel and has a diameter of $15 \mathrm{~mm}$. Both the ball and the disk could be rotated independently to give a range of slide-to-roll ratios. The slide-to-roll ratio is defined as $2\left(U_{\mathrm{d}}-U_{\mathrm{b}}\right) /\left(U_{\mathrm{d}}+U_{\mathrm{b}}\right)$, where $U_{\mathrm{d}}$ and $U_{\mathrm{b}}$ are the surface velocities of the disk and the ball, respectively. The test conditions are summarized in Table 2 .

Prior to each of the tests, all specimens were cleaned with acetone in an ultrasonic bath and dried. Fresh grease was uniformly smeared on the disk to a thickness of 0.1 to $0.2 \mathrm{~mm}$, and no additional lubricant was supplied during the test. Both in film thickness

Table 1 Greases used

\begin{tabular}{llll}
\hline Grease & Thickener type & Base oil and viscosity $\left(\mathrm{mm}^{2} / \mathrm{s}\right)$ & Penetration \\
\hline L1 & 12 mass\% lithium 12-hydroxystearate & Poly-alpha-olefin (PAO) 60 & 291 \\
L2 & 12 mass\% lithium 12-hydroxystearate & Poly-alpha-olefin (PAO) 400 & 386 \\
L3 & 20 mass\% lithium 12-hydroxystearate & Polyol ester (POE) 32 & 221 \\
D & 12 mass\% di-urea & Polyol ester (POE) 32 & 280 \\
\hline
\end{tabular}

All viscosities are at $313 \mathrm{~K}$ 


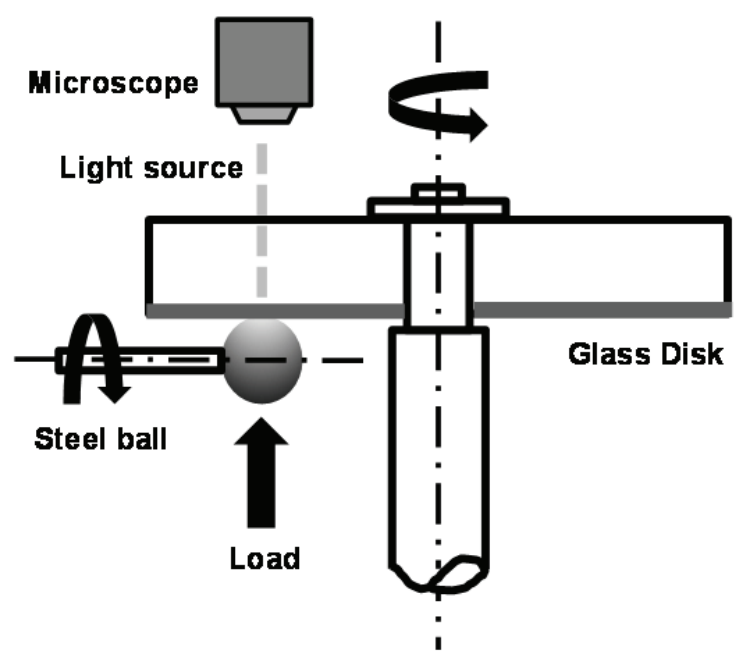

Fig. 1 Ball-on-disk test rig

measurement and track pattern observation, the tests were made from the lowest speed to the highest entrainment speed.

The central film thickness was measured using the same test rig, using a white light source. A high speed camera was connected to the microscope and recorded the behavior of grease flow in the vicinity of an EHL contact. SLIM, the spacer layer imaging method [19] was used to determine the film thickness for a range of speed. The RGB values for each pixel in the images were converted into hue value, from which the film thickness was determined.

A monochrome CCD camera was used to get high quality pictures of tracks on the disk specimens. At the end of each test, the pictures were captured immediately at five locations on the disk including upstream, inlet, center, outlet and downstream of the EHL conjunction. The upstream and downstream images were taken at 4.5 times the Hertzian contact diameter upstream and downstream from the contact center, respectively.

Another series of tests with the same test lubricants and test conditions were conducted by using a pair of scoops. The scoops were pressed up against the underside of the disk near the inlet contact zone to channel grease back to the contact zone, as shown in Fig. 2.

Table 2 Test conditions

\begin{tabular}{ll}
\hline Test conditions & \\
\hline Entrainment speed & $1-500 \mathrm{~mm} / \mathrm{s}$ \\
Temperature & $298 \mathrm{~K}$ \\
Normal load & $10 \mathrm{~N}$ \\
Maximum Hertzian pressure & $0.87 \mathrm{GPa}$ \\
Hertzian contact radius & $98.7 \mu \mathrm{m}$ \\
Track radius & $37.5 \mathrm{~mm}$ \\
\hline
\end{tabular}

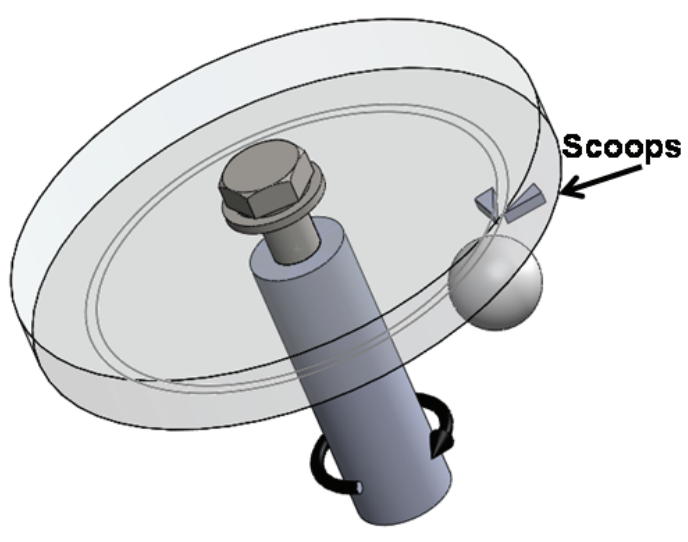

Fig. 2 Ball-on-disk configuration with scoops

This means they erased the track pattern in the inlet regime. For this reason, only the track pattern pictures at the center, outlet and downstream of the conjunction were taken in the tests with the scoops. In this study, inlet and downstream pictures were used in following discussion. Also, the characteristics of the grease track pattern and the variation trend with entrainment speed show no significant difference between the positive and negative slide-to-roll ratios in our test conditions. Therefore, only the data with a slide-to-roll ratio of 0.4 are shown in this paper.

\subsection{Viscosity measurement}

Rheological measurements were carried out using a Paar Physica MCR 301 rheometer, which controls both stress and shear. The rotating part was supported by air bearings. A cone-on-plate with a diameter of $25 \mathrm{~mm}$, an angle of $2^{\circ}$ and a gap size of $0.1 \mathrm{~mm}$ was used for this study. Viscous flow measurements in the shear rate range of $10^{-3}$ to $10^{3} \mathrm{~s}^{-1}$ were carried out to measure the apparent viscosity as a function of the shear rate. The tests were repeated at least three times with pre-sheared samples at $298 \mathrm{~K}$, and the results presented here are the average values. As is generally known, the greases show high apparent viscosity under low shear rates, and the apparent viscosity reduces with the shear rate to approach to that of the base oil. Table 3 shows the apparent viscosity of the greases at low and high representative shear rates of $1 \mathrm{~s}^{-1}$ and $1000 \mathrm{~s}^{-1}$. Table 4 shows the viscosity of the corresponding base oils.

Table 3 Apparent viscosity of the greases at $298 \mathrm{~K}$

\begin{tabular}{lllll}
\hline Grease & L1 & L2 & L3 & D \\
\hline$\eta(\mathrm{Pa} \cdot \mathrm{s})$ at $1 \mathrm{~s}^{-1}$ & 367 & 101 & 1028 & 3280 \\
$\eta(\mathrm{Pa} \cdot \mathrm{s})$ at $1000 \mathrm{~s}^{-1}$ & 0.33 & 1.03 & 0.27 & 0.085 \\
\hline
\end{tabular}


Table 4 Apparent viscosity of the base oils at $298 \mathrm{~K}$

\begin{tabular}{llll}
\hline Base oil & PAO60 & PAO400 & POE32 \\
\hline$\eta(\mathrm{Pa} \cdot \mathrm{s})$ & 0.12 & 0.88 & 0.06 \\
\hline
\end{tabular}

\section{Results}

\subsection{Film thickness}

Figure 3 shows the variation of the central film thickness with entrainment speed in the tests with scoops and without scoops. In the tests with scoops, shown in Fig. 3(a), the film thickness is proportional to speed to the power of about 0.7 at higher speed, showing the typical fully-flooded EHL behavior. The film is slightly thicker than the theoretical film thickness

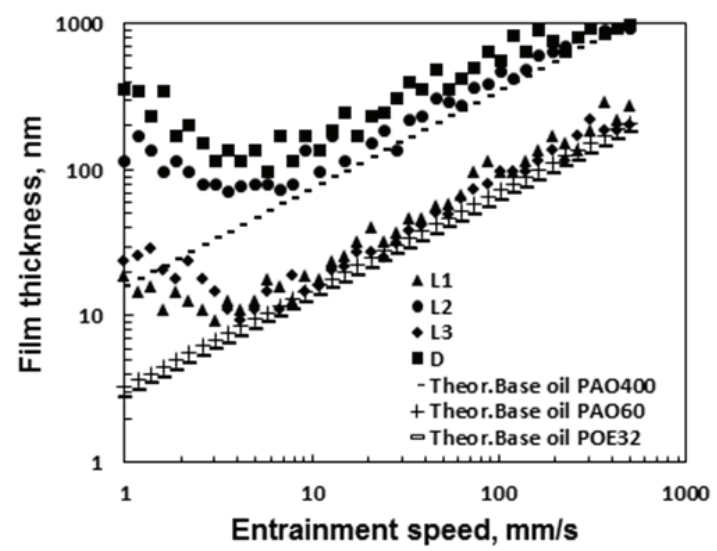

(a)

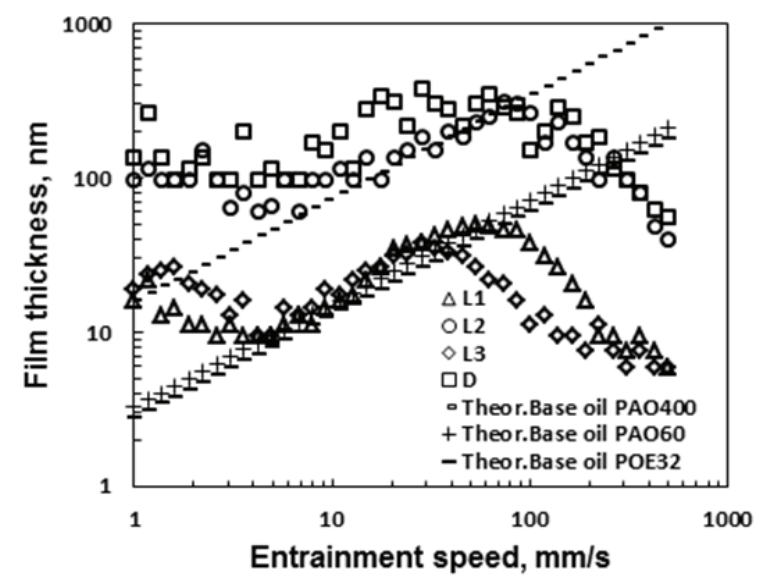

(b)

Fig. 3 Variation of central film thickness with entrainment speed; (a) in the tests with scoops, and (b) in the tests without scoops for the corresponding base oils except for the grease D. The larger film thickness for the greases L1, L2 and L3 is due to higher apparent viscosity than the base oils as shown in Tables 3 and 4, while the significantly thicker film with the grease D is caused by the thickener as will be shown in the next section. In the lower speed range, the film thickness increases to form a V-curve. This deviation from the straight line in the lower speed range has been reported by many researchers, and is explained most simply by the increase in the apparent viscosity at low shear rates [20].

In the test without the scoops, shown in Fig. 3(b), the film thickness variation is essentially the same in the low to middle speed ranges. At higher entrainment speed, the film thickness deviates from the straight lines to lower values, indicating the occurrence of the inlet starvation. The corresponding starvation speed for the greases L3 and D are approximately $28 \mathrm{~mm} / \mathrm{s}$ and 18 $\mathrm{mm} / \mathrm{s}$, which are smaller than $54 \mathrm{~mm} / \mathrm{s}$ and $74 \mathrm{~mm} / \mathrm{s}$ for the greases L1 and L2, respectively. It is likely that the starvation occurs at smaller entrainment speed with greases of lower viscosity base oil, which is usually not the case with fluid lubricants. This will be discussed in Chapter 4.

\subsection{Features of track patterns}

Figure 4 shows the images of the downstream track on the disks for three entrainment speeds in the tests without scoops. Figure 5 shows the downstream images for the entrainment speed of $225.4 \mathrm{~m} / \mathrm{s}$ in the test with the scoops. The arrows in all images used in this study show the direction of motion of the specimen surfaces if not mentioned otherwise. The images include the track at the center, and finger-like patterns on both sides of the track. The fingers are formed by the outlet cavity, and consist of branched ribs of grease separated by air spaces. On the tracks is seen the deposition of the greases in some cases. In contrast with fluid lubricants, which are believed to flow more easily to replenish the track, greases stays at the sides of the track due to its rather immobile nature under low shear rates. This persistence of the grease may serve to lubricate in subsequent passes, as will be observed in the upstream of the conjunction.

The appearances of the downstream images have different features depending on grease type and test conditions. The lithium soap greases L1, L2 and L3 show finger flow patterns, although the patterns are rough in the low entrainment speed of $1 \mathrm{~mm} / \mathrm{s}$ and finer at higher speeds. In Fig. 4, the patterns at the high speed of $225.4 \mathrm{~mm} / \mathrm{s}$ are less regular and distinct in starved conditions without the scoops than those with the scoops shown in Fig. 5. This will be called "finger-loss" in what follows. On the contrary, the grease D shows completely different track patterns with little or very vague finger patterns but heavy deposition of the grease on the center of the track with inhomogeneous and blurry boundary at the edges of the track. 

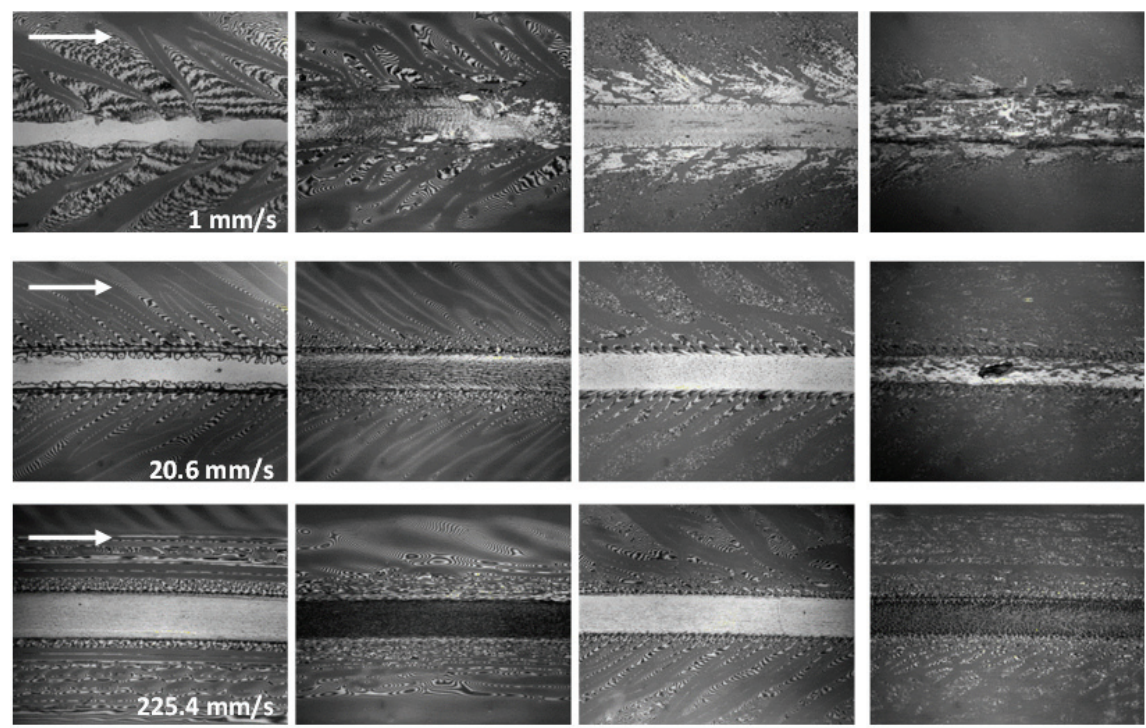

$-100 \mu \mathrm{m}$

L1

L2

L3

D

Fig. 4 Downstream images at three entrainment speeds in the test without scoops

Figure 6 shows the pictures of the inlet taken after the tests without the scoops. The EHL conjunction is at the right end in each of the pictures. The darker part lying upstream of the conjunction is the grease being entrained, and its boundary with remained track pattern in further upstream clearly shows the shape of the inlet meniscus. It can be seen from these pictures that the meniscus approaches the contact circle at higher the entrainment speed for all the greases, as observed by Pemberton and Cameron [7], and Aström et al. [17]. The inlet starvation is clearly seen at the speed of 225.4 $\mathrm{mm} / \mathrm{s}$ with all the greases.

Closer examination of the inlet pictures reveals that, the fingers present in upstream, shown in Fig. 4, appear to change little as they approach the meniscus, which suggests the greases at the track sides do not substantially flow back before the tracks come closer to the contact with the counter surface again. On the other hand, the meniscus looks different for different greases. The meniscuses for the greases L1 and L2 exhibit simpler fluid-like shape whereas those for the greases L3 and D show more irregular shape for all the speeds shown. This may be because of the fluidity of the grease when they are merging into the meniscus at smaller shear rate.

\subsection{Finger interval and inlet distance}

The images given in the above section show the complexity of the grease track pattern. Among a number of the features of the tracks, two geometrical quantities are chosen here to characterize the track pattern and the inlet starvation.

The first one is the average interval between the fingers, which is called the finger interval $\lambda$ in what follows. Because the fingers are periodic wake formed at the diverging outlet with the cavitation, and are branched and finely divided near the contact track with smaller gap between the surfaces, a representative interval for the wake appears in non-branched part, which is some distance from the track. Therefore, the

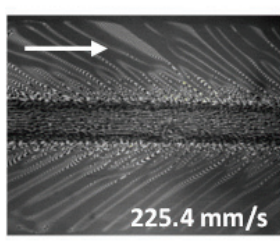

$-100 \mu \mathrm{m}$

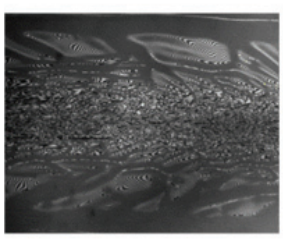

L2

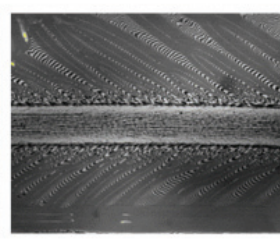

L3

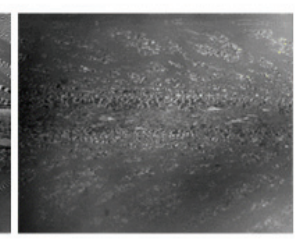

D

Fig. 5 Downstream images at $225.4 \mathrm{~mm} / \mathrm{s}$ in the test with scoops 

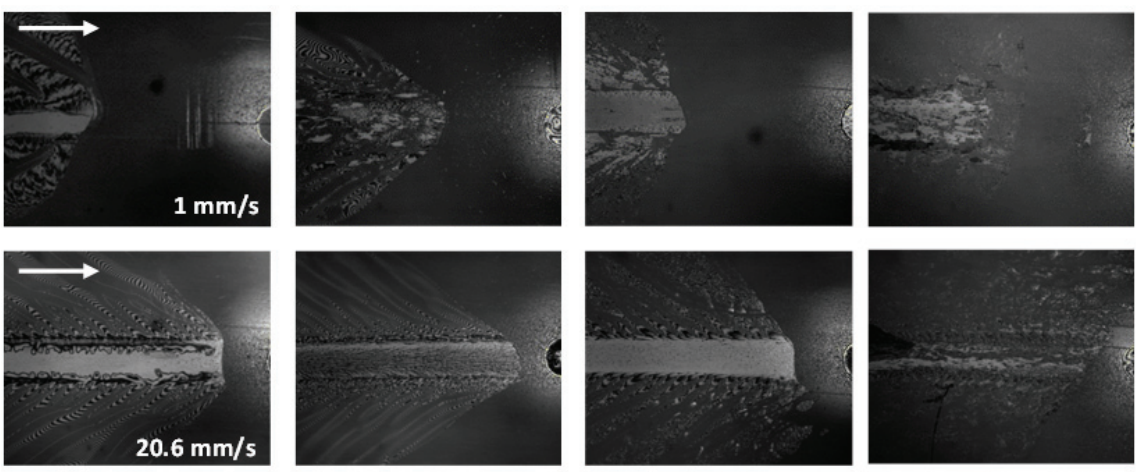

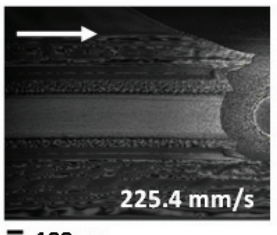

$-100 \mu \mathrm{m}$

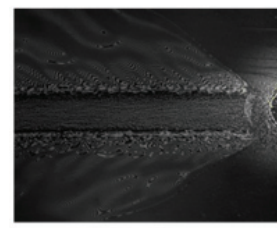

L

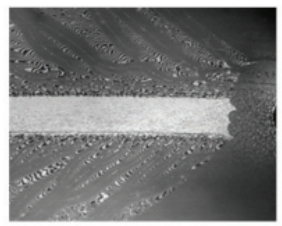

L3

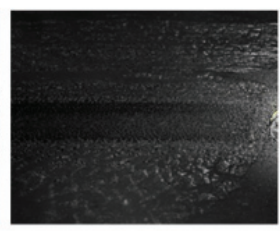

D

Fig. 6 Inlet images during the tests without scoops at three entrainment speeds

finger interval $\lambda$ is defined here as the average interval measured perpendicular to the average tilt angle at a position 4 to 5 times the Hertzian contact radius apart from the center of the track, as shown in Fig. 7.

The finger interval $\lambda$ determined for the tests with the three greases with lithium soap thickeners L1, L2, and L3 are shown in Fig. 8. Although the values are scattered, the finger interval data all present a decreasing trend, and they have similar values regardless of the grease at the middle speed range where the starvation starts to occur. This indicates that the finger interval depends on the entrainment speed rather

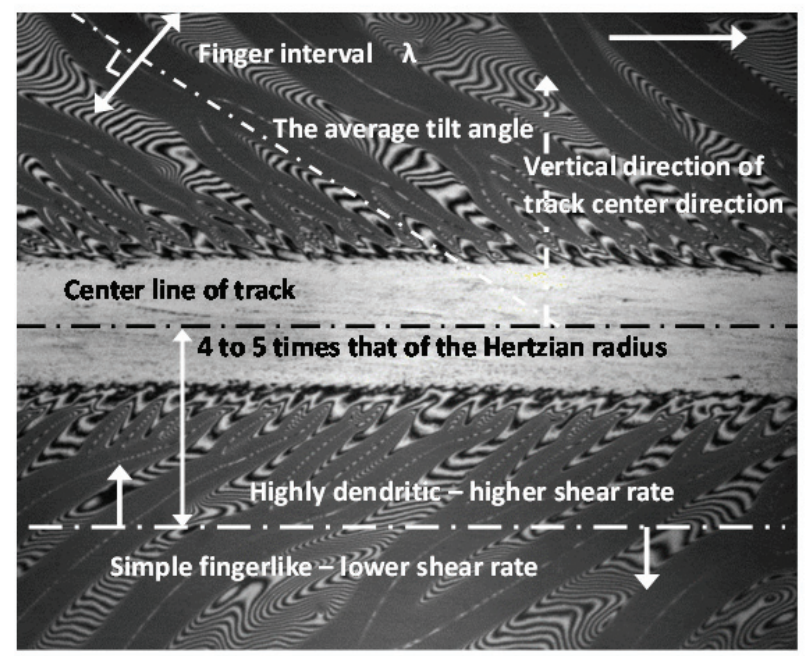

$-100 \mu \mathrm{m}$

Fig. 7 Definition of finger interval in downstream image than the film thickness.

The other parameter introduced in this paper is the inlet distance $S$, which is defined as the distance between the front edge of the contact circle and the meniscus. The result is shown in Fig. 9. As predicted from the pictures in Fig. 6, the inlet distance $S$ decreases with the entrainment speed.

\section{Discussion}

\subsection{Starvation and grease properties}

For the four greases used in this study, it has been shown in the film thickness measurement that the starvation occurs at smaller entrainment speed with greases of lower viscosity base oil. The observation of

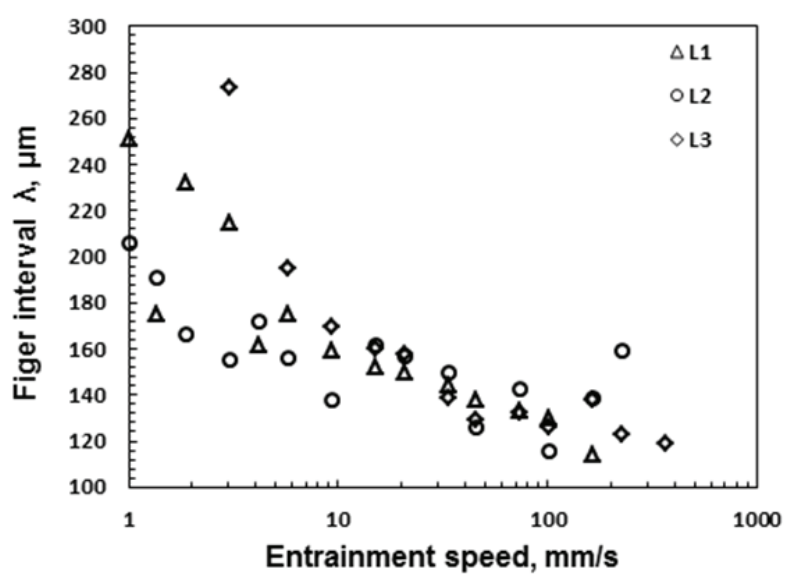

Fig. 8 Variation of finger interval with entrainment speed in the test without scoops 


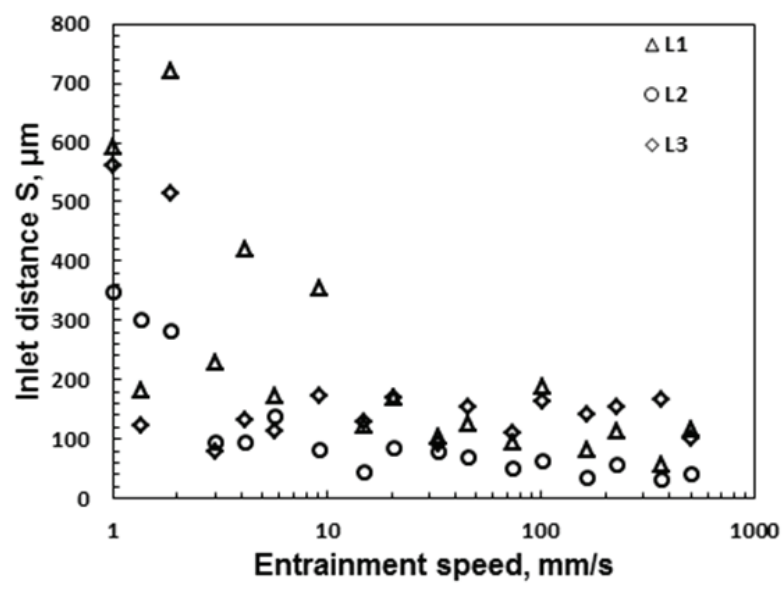

Fig. 9 Variation of inlet distance with entrainment speed in the test without scoops

the inlet meniscus also supported this trend. However, this is usually not the case with fluid lubricants because fluids with lower viscosity can flow back to the track more quickly [8]. The flow behavior at the inlet meniscus shown in Fig. 6 demonstrates that the greases L1 and L2 have fluidity to be supplied to the inlet meniscus more easily than the other greases although L1 and L2 contain higher viscosity base oils. This implies that the viscosity property of greases rather than base oil viscosity play an important role in the resupply of the grease; lower apparent viscosity at low shear rates helps the greases to be supplied to the contact, which should work to maintain the fully flooded condition at higher speeds.

This is clearly seen in Fig. 10 in which the measured apparent viscosities at two representative shear rates are plotted against the starvation speed in the tests without the scoops. The inlet starvation occurs at lower entrainment speed with the greases with higher apparent

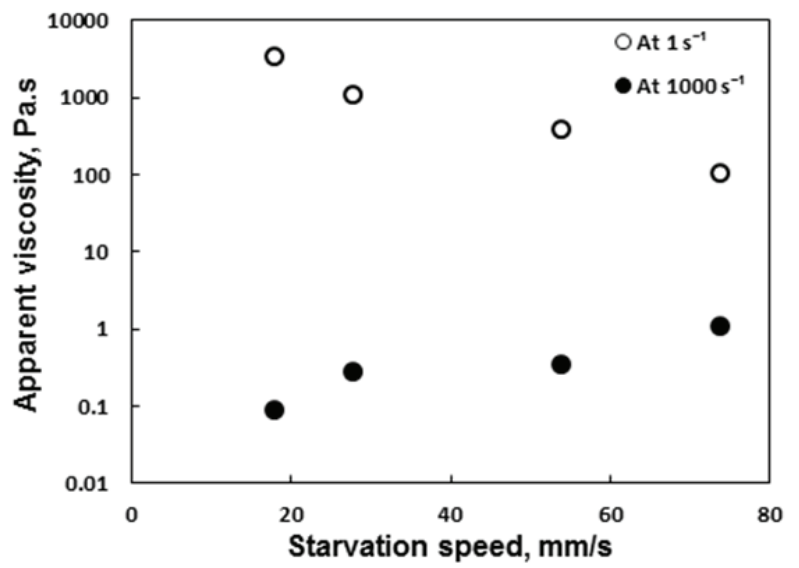

Fig. 10 Relationship between starvation speed and apparent viscosity at two shear rates viscosity. The figure may also suggest that higher viscosity at high shear rate has promoted the film formation, although it is a mere coincidence that the greases with lower base oil viscosity has higher apparent viscosity under low shear rates in this study. Whether it is true or not, the present conclusion here for the starvation is the availability of the grease depends on the apparent viscosity at low shear rate.

\subsection{Track patterns}

It has been shown in the present study that the downstream flow patterns are preserved more or less, and they depend on the grease and the speed. Their patterns should depend on a fluid dynamic action to form wake at the outlet, including formation of the outlet cavity. This in turn may depend on rheological properties of greases, outlet diverging shape, and surface tension.

The present observation has revealed that the finger interval decreases with the entrainment speed, and their values for the same speed are not very different for different greases that provide different film thickness. This suggests that the formation of the periodic finger pattern depends not on the detailed dynamics to generate hydrodynamic pressure but on the speed of the greases to pass by the conjunction.

It should be noted that the finger-like pattern at the sides of the track is formed even in the starved conditions, and the finger-loss occurs at a higher speed than the starvation speed, as shown in Fig. 11. The formation of the finger-like pattern is the evidence for the fluid dynamic action to be working. Nevertheless, when the grease in the inlet zone is depleted at higher speed, it becomes increasingly difficult to maintain the EHL film, and the outlet cavity is destroyed and the finger track pattern cannot be formed.

From the present result, it can be said that the average finger interval itself does not provide information regarding the replenishment capability of the greases. However, the pattern of the finger branches

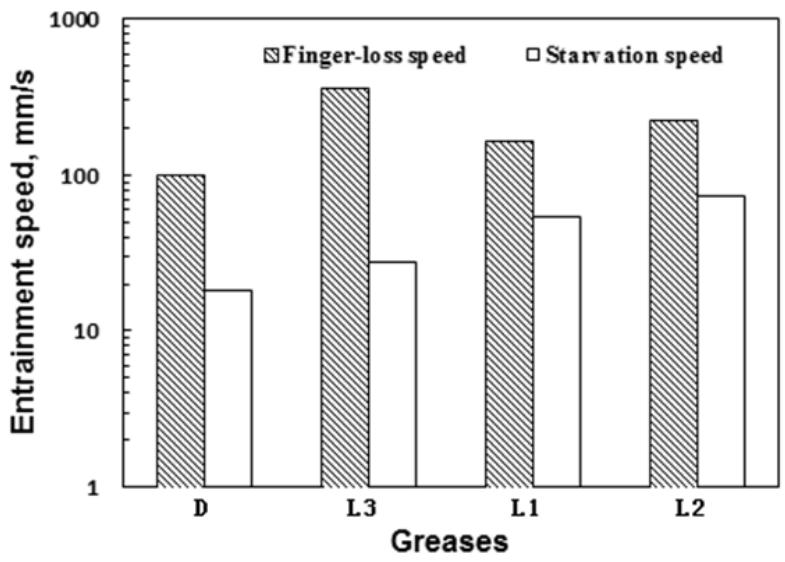

Fig. 11 Finger-loss speed and starvation speed for the four grease 
and the detailed structures formed on the side ridges may contain information as to rheological properties of greases. Also, the clarity or contrast of the finger pattern images reflects the geometrical shape of the fingers, and may depend on greases. From Fig. 4, it appears that the greases with lower apparent viscosity under low shear rate show clearer patterns. These characteristics of the finger patterns and their relation with the grease rheology remain to be investigated in the future work.

Another particular aspect of the grease track pattern is the deposition of greases on the track, particularly with the urea grease D. It is likely that it provides rather solid lubrication at higher speeds when the fluid starvation occurs. The phenomena of grease deposition have already been found in other works [2,12], although there still is little understanding as to how they are deposited and how their appearance is determined. The non-uniform patterns are due to deposition of thickener lumps, and therefore show different features depending on the grease. Further work is necessary to characterize the deposited patterns.

\section{Conclusions}

The conclusions drawn from this study are as follows.

1. The features of grease track pattern changes with grease type and test conditions.

2. The average interval between fingers decreases with increasing entrainment speed and is mainly determined by entrainment speed.

3. Starvation and finger-loss occur at higher entrainment speeds with all the tested greases. The starvation speeds are lower than the finger-loss speeds

4. The apparent viscosity of the greases at low shear rate in the inlet zone has a great impact on the resupply of the greases and the starvation speed.

\section{References}

[1] Lugt, P. M., "A Review on Grease Lubrication in Rolling Bearings," Tribology Transactions, 52, 4, 2009, 470-480.

[2] Cann, P. M., "Grease Lubrication of Rolling Element Bearings - Role of the Grease Thickener," Lubrication Science, 19, 3, 2007, 183-196.

[3] Hokao, M., Inami, N., Watabe, E., Yokouchi, A. and Sugimura, J., "A Study of the Structure Formed by Thickeners of Greases Using Atomic Force Microscope," Tribology Online, 8, 2013, 76-82.

[4] Wikström, V. and Jacobson, B., "Loss of Lubricant From Oil-Lubricated Near-Starved Spherical Roller Bearings," Journal of Engineering Tribology, 211, 1, 1997, 51-66.

[5] Scarlett, N. A., "Use of Grease in Rolling Bearings," Proc. Instn. Mech. Engrs., 182, 1967, 585-624.
[6] Wedeven, L. D., Evans, D. and Cameron, A., "Optical Analysis of Ball Bearing Starvation," Journal of Tribology, 93, 3, 1970, 349-361.

[7] Pemberton, J. and Cameron, A., "A mechanism of Fluid Replenishment in Elastohydrodynamic Contacts," Wear, 37, 1, 1976, 185-190.

[8] Chiu, Y. P., "An Analysis and Prediction of Lubricant Film Starvation in Rolling Contact Systems," ASLE Transactions, 17, 1, 1974, 37-41.

[9] Jacod, B., Pubilier, F., Cann, P. M. and Lubrecht, A. A., "An Analysis of Track Replenishment Mechanisms in the Starved Regime," Lubrication at the Frontier, Tribology Series, 36, D. Dowson et al eds., Elsevier, 1999, 483-492.

[10] Gershuni, L., Larson, M. G. and Lugt, P. M., "Lubricant Replenishment in Rolling Bearing Contacts," Tribology Transactions, 51, 5, 2008, 643-651.

[11] Cann, P. M., "Starvation and Reflow in a Grease-Lubricated Elastohydrodynamic Contact," Tribology Transactions, 39, 3, 1996, 698-704.

[12] Cann, P. M., "Starved Grease Lubrication of Rolling Contacts," Tribology Transactions, 42, 4, 1999, 867-873.

[13] Kaneta, M., Ogawa, T., Takubo, Y. and Naka, M., "Effects of a Thickener Structure on Grease Elastohydrodynamic Lubrication Films," Proc. IMechE, Pt. J, Journal of Engineering Tribology, 214, 2000, 327-336.

[14] Kimura, H., Imai, Y. and Yamamoto, Y., "Study on Fiber Length Control for Ester-Based Lithium Soap Grease," Tribology Transactions, 44, 3, 2001, 405-410.

[15] Yokouchi, A., Hokao, M. and Sugimura, J., "Effects of Soap Fiber Structure on Boundary Lubrication of Lithium Soap Greases," Tribology Online, 6, 4, 2011, 219-225.

[16] Oikawa, E., Inami, N., Hokao, M., Yokouchi, A. and Sugimura, J., "Bearing Torque Characteristics of Lithium Soap Greases with Some Synthetic Base Oils," Proc. IMechE, Pt. J., Journal of Engineering Tribology, 226, 6, 2012, 575-583.

[17] Åström, H., Östensen, J. O. And Höglund, E., "Lubricating Grease Replenishment in an Elastohydrodynamic Point Contact," Journal of Tribology, 115, 3, 1993, 501-506.

[18] Otsu, T., Tanaka, H. and Sugimura, J., "Initiation and Growth of Gaseous Cavity in Concentrated Contact in Various Surrounding gases," Tribology International, 53, 2012, 68-75.

[19] Cann, P. M., Spikes, H. A. and Hutchinson, J., "The Development of a Spacer Layer Imaging Method (SLIM) for Mapping Elastohydrodynamic Contacts," Tribology Transactions, 39, 4, 2008, 915-921.

[20] Kimura, Y., Endo, T. and Dong, D., "EHL with Grease at Low Speeds," Advanced Tribology, Springer Berlin Heidelberg, 2010, 15-19. 